\title{
Identification of Potent VEGF Inhibitors for the Clinical Treatment of Glioblastoma, A Virtual Screening Approach
}

\author{
Mohini Yadav ${ }^{1}$, Ravina Khandelwal ${ }^{1}$, Urvy Mudgal ${ }^{1}$, Sivaraj Srinitha ${ }^{1}$, Natasha \\ Khandekar ${ }^{1}$, Anuraj Nayarisseri1 ${ }^{12 *}$, Sugunakar Vuree ${ }^{3}$, Sanjeev Kumar Singh ${ }^{4 *}$
}

\begin{abstract}
Vascular endothelial growth factor (VEGF) expression could be found in all glioblastomas. VEGF takes part in numerous changes including the endothelial cell proliferation, the vasculature of solid tumor: its survival invasion, and migration, chemotaxis of bone marrow-derived progenitor cells, vasodilation and vascular permeability. VEGF inhibition can be a smart therapeutic strategy because it is extremely specific and less toxic than cytotoxic therapy. To establish better inhibition of $V E G F$ than the current inhibitors, present study approach is by molecular docking, virtual screening to illustrate the inhibitor with superior affinity against $V E G F$ to have a cautious pharma profile. To retrieve the best established and high-affinity high affinity molecule, Molegro Virtual Docker software was executed. The high-affinity scoring compounds were subjected to further similarity search to retrieve the drugs with similar properties from pubchem database. The completion of virtual screening reveals that PubChem compound SCHEMBL1250485 (PubChem CID: 66965667) has the highest affinity. The study of the drug-likeness was verified using OSIRIS Property Explorer software which supported the virtual screened result. Further ADMET study and drug comparative study strongly prove the superiority of the new established inhibitor with lesser rerank score and toxicity. Overall, the new inhibitor has higher potential to stop the expression of $V E G F$ in glioblastoma and positively can be further analysed through In vitro studies.
\end{abstract}

Keywords: $V E G F$ - glioblastoma- virtual screening- molecular docking- ADMET profiling- drug likeness verification

Asian Pac J Cancer Prev, 20 (9), 2681-2692

\section{Introduction}

The term 'glioma' comprehends all tumors encompassing the glial cell origin, including astrocytoma grades I, II, III and IV (Schwartzbaum et al., 2006). Unfortunately, grade 4 which is Glioblastoma multiforme (GBM), is the most aggressive and also the most common in humans. This brain tumor drew the noteworthy attention of scientists and doctors all over the world as patients with GBM die within a year having no scope of long term survival. However, the research evaded progressively high and complex attempts at therapy over the past half-century (Holland, 2000) which still accounts for 12 percent to 15 percent of all intracranial tumors and 50 to 60 percentage of astrocytic tumors with an annual incidence rate of 5.26 per 100,000 population new diagnoses per year (Rosell et al., 2008, Omuro and DeAngelis, 2013). The survival rate is almost similar in various patients and also not influenced by the patient's sex, the grade of tumor and the extent of surgery (Simpson and Platts, 1976). But the patients diagnosed with GBM in the early ages of 40 to 50 years show higher 5-year overall survival rates (Stupp et al., 2009). Glioblastoma shows multiple complex character types as its name has multiple forms which is one of the reasons for the resistance of GBM to therapeutic intervention. GBM is excessively showing regions of necrosis and haemorrhage (Holland, 2000). This lethal GBM reappears in nearly all patients and no effective generalized treatment exists for the recurrent diseases up till now. Therefore, advances in the GBM treatment and drugs in all clinical and scientific aspects are urgently needed (Lee et al., 2017). A case study of 153 recurrent glioblastoma patients in 2014 summarises the approval by the FDA on the most effective drug bevacizumab, which can increase the survival up tomonths, which $38 \%$ and combined treatment of bevacizumab with lomustine can increase the survival rate up to $59 \%$. This data indicates the need for new and more effective drugs and treatment options for the patients of recurrent GBM (Mehta and Brem et al., 2014). Glioblastoma multiform

${ }^{I}$ In silico Research Laboratory, Eminent Biosciences, ${ }^{2}$ Bioinformatics Research Laboratory, LeGene Biosciences Pvt Ltd., Indore - 452 010, Madhya Pradesh, ${ }^{3}$ Department of Biotechnology, Lovely Faculty of Technology and Sciences, Division of Research and Development, Lovely Professional University, Phagwara, Punjab-144411, ${ }^{4}$ Computer Aided Drug Designing and Molecular Modeling Lab, Department of Bioinformatics, Alagappa University, Karaikudi-630 003, Tamil Nadu, India. *For Correspondence: anuraj@eminentbio.com,skysanjeev@gmail.com 
has two noticeable features that are angiogenesis and tumorigenesis. One a vascular factor that can connect both the noticeable features (Greenberg et al., 2005). Angiogenesis controls the growth and synthesis of all the tumors by forming new blood vessels from pre-existing vessels (Folkman, 1971). Tumor angiogenesis is coordinated by a synchronized increase in the expression of genes, including $V E G F, I L-8$ and $I L-6$, acidic as well as basic fibroblast growth factor (FGF), angiopoietins and the hypoxia-inducible factor 1 alpha (HIF-1 $\alpha$ ), with downregulation of endogenous angiogenesis inhibitors such as: endostatin, thrombospondins, interferon and angiostatin (Hanahan and Folkman, 1996). VEGF is a supreme common denominator required for tumor angiogenesis and pathogenesis (Ferrara et al., 2003).

The $V E G F$ and its receptors are expressed in GBM and angiogenesis are involved in nearly every stage of cancer, from the first stage of cancer formation to the last stage of distant metastasis. VEGF inhibition can be a smart therapeutic strategy because it is extremely specific and less toxic than cytotoxic therapy. $V E G F$ inhibitors offer a means to control a heterogeneous tumor population by influencing a relatively homogeneous endothelial population. Therefore the current study ended with the better inhibitor for $V E G F$ by using docking and drug designing to ascertain that the new compound plays a significant role in upsurge the survival of the GBM patients.

\section{Materials and Methods}

\section{Selection of inhibitor}

Innitiating the inhibitor selection, existing inhibitors of $V E G F$ that target GBM were selected from several literatures. The availability of total numbers of established inhibitors were 16, selected for further observations. Some inhibitors were lacking their 3D structures. The 3D structures of all those compounds were modelled using Marvin Sketch and was saved in 3D SDF format (Ali et al., 2019; Khandelwal et al., 2018). All the 16 inhibitors accordingly having Pubchem ID with 3D structures and prepared 3D structures are present in the Table1.

\section{Protein and Ligand preparation}

The crystal structure of the target protein, an extracellular domain of $V E G F$ was retrieved from protein Data Bank (PDB) with PDB ID: 3V2A (Brozzo et al., 2012). Few amino acids side-chain atoms were missing in the receptor structure $3 \mathrm{~V} 2 \mathrm{~A}$. A reconstruction of the whole side-chain was performed using Modellar software. 21 side-chain atoms were added in the present $3 \mathrm{D}$ structure and the energy minimization was performed in vacuo with the Gromos 96 algorithm using 43B1 parameters set, without reaction field (Akare et al., 2014; Babitha et al., 2018; Bandaru et al., 2013; Bandaru et al., 2014). After adding the missing side-chain atoms the energy was $-11310.358 \mathrm{KJ} / \mathrm{Mol}$ which shows better stability to the $3 \mathrm{D}$ structure of $V E G F$. Hence the same was used for the molecular docking studies. The inhibitors accomplishing a Pubchem ID were retrieved the 3D conformer of inhibitors. Further preparation of ligand was preceded by taking the $3 \mathrm{D}$ structure of all those compound embedded in LegPrep module, an application of Schrodinger suite (Schrodinger. LLC, New York, NY) and were optimized through OPLS 2005 force field algorithm (Bandaru et al., 2015a; Bandaru S et al., 2015b; Babitha et al., 2015; Chandrakar et al., 2013; Divya Jain et al., 2019; Dunna et al., 2015a; Gudala, et al., 2015; Majhi et al., 2018). The prepared ligands were saved in a single SDF file for further docking studies (Bandaru et al., 2016; Basak et al., 2016a; Dunna et al., 2015b; Mendonça-Junior et al., 2019; Nasr et al., 2015).

\section{Molecular docking}

The molecular docking study was performed by using Molegro Virtual Docker (MVD) which is unified with high potential Piece Wise Linear Potential (PLP) and MVD scoring function (Bandaru et al., 2017a; Basak et al., 2016b; Gutlapalli et al., 2015; Kelotra et al., 2014; Natchimuthu et al., 2016; Nayarisseri et al., 2018; Sharda et al., 2019; Padmini et al., 2019). The pre-prepared 16 ligands were saved in one single SDF file. PDB file of target protein consisting of pre-existing ligands were removed. It was then prepared further by detecting cavities. A cavity, namely the fourth cavity bearing a volume of $13.825 \mathrm{Ao}$ was targeted for further procedure of docking with ligands. Docking process possessed the of maximum iteration of 1,500, maximum population size 50 , Grid solution 0.2 ; having a binding affinity, the protein and ligands were evaluated on the following conformation of the Internal Electrostatic interaction (Internal ES), sp2-sp2 torsions, and internal hydrogen bond interaction. Binding site defined the first cavity according to the highest volume. The post dock study involved energy minimization and $\mathrm{H}$-bond optimization. After docking, to minimize the complex energy of ligand-receptor interaction, the Nelder Mead Simplex Minimization (using non grid force field and H-bond directionality) was used (Sahila et al., 2017; Bandaru et al., 2017b; Kelotra et al., 2014; Monteiro et al., 2018; Nasr et al., 2015).

\section{Virtual screening}

With reference to our target query compound Cediranib, a similarity search was executed to obtain the best compound having a greater affinity other than any established drugs against the PubChem database developed by NIH, which is one of the public chemical repository containing 93 million chemical compounds database(Sinha et al., 2014; Sinha et al., 2018; Trishang et al., 2019; Vuree et al., 2013; Nayarisseri et al., 2019; Sharma et al., 2018; Shaheen et al., 2015; Shameer et al., 2017). The filtrations property parameter set by component rule of Lipinski's rule of five at threshold $>=95 \%$ were done against NCBI's PubChem compound database (Nayarisseri et al., 2015; Sharda et al., 2017; Khandelwal et al., 2018; Khandekar et al., 2016; Palak S et al., 2019; Patidar K et al., 2019; Patidar, K et al., 2016; Sinha et al., 2015; Sahila et al., 2015). These compounds were with the same procedure, preceded for Molecular Docking with the target protein $V E G F$ to find the compound having surpassed affinity. 
Table 1. Established VEGF Inhibitors with Pubchem CID

\begin{tabular}{|c|c|c|c|c|c|c|c|}
\hline SI & Inhibitor & Pub Id & $\mathrm{Mw}(\mathrm{g} / \mathrm{mol})$ & HBD & HBA & $\mathrm{X} \log \mathrm{P}$ & Reference \\
\hline 1 & Semaxanib (SU5416) & 5329098 & 238.29 & 2 & 1 & 2.5 & (Vajkoczy et al., 1999) \\
\hline 2 & Vatalanib & 151194 & 346.818 & 1 & 4 & 4.5 & (Gerstner et al.,2009) \\
\hline 3 & Sunitinib & 5329102 & 398.482 & 3 & 4 & 2.6 & (Gerstner et al.,2009) \\
\hline 4 & Sorafenib & 216239 & 464.829 & 3 & 7 & 4.1 & (Gerstner et al.,2009, Loges) \\
\hline 5 & Cediranib(AZD2171) & 9933475 & 450.514 & 1 & 7 & 4.9 & (Batchelor et al., 2007a; Gerstner et al.,2009) \\
\hline 6 & CEP-7055 & 9936664 & 525.649 & 1 & 5 & 4.5 & (Jones-Bolin et al., 2006a) \\
\hline 7 & XL-184 & 25102847 & 501.514 & 2 & 7 & 5.4 & $\begin{array}{l}\text { (Gerstner et al.,2009; Zhang et al., 2010; Hernandez- } \\
\text { Pedro et al., 2013) }\end{array}$ \\
\hline 8 & Nelfinavir & 64143 & 567.789 & 4 & 6 & 5.7 & (Kast et al., 2013; Mangraviti et al., 2017) \\
\hline 9 & AEE788 & 10297043 & 440.595 & 2 & 5 & 4.6 & (Gerstner et al., 2009; Reardon et al., 2012) \\
\hline 10 & Amprenavir & 65016 & 505.63 & 3 & 8 & 2.9 & (Pore et al., 2006) \\
\hline 11 & Acriflavine & 443101 & 468.989 & 4 & 6 & -- & (Mangraviti et al., 2017) \\
\hline 12 & Pazopanib & 10113978 & 437.522 & 2 & 8 & 3.1 & (Gerstner et al., 2009; Taylor and Gerstner, 2013) \\
\hline 13 & Vandetanib or ZD6474 & 3081361 & 475.362 & 1 & 7 & 4.9 & $\begin{array}{l}\text { (Gerstner et al., 2009; Taylor and Gerstner, 2013; } \\
\text { Jawhari et al., 2016) }\end{array}$ \\
\hline 14 & Pegdinetanib or СТ322 & 86278317 & 694.754 & 6 & 15 & -5.7 & (Mamluk et al., 2010) \\
\hline 15 & Sodium butyrate & 5222465 & 110.088 & 0 & 2 & -- & (Sawa et al., 2002) \\
\hline 16 & Trichostatin A & 444732 & 302.374 & 2 & 4 & 2.7 & (Sawa et al., 2002) \\
\hline
\end{tabular}

\section{Drug - Drug comparative study}

The unnamed complex structure was retrieved from the established drug docking result. It was cleaned by removing all the ligands, constraints, and cavities except the protein which is eventually imported with the best posed inhibitor and exported as best drug docked file in SDF format (Praseetha et al., 2016a; Praseetha et al., 2016b; Rao et al., 2010). The complex structure was retrieved from the virtual docking result and the procedure was repeated. The excel sheet was prepared to check all the affinities, hydrogen interactions, steric energy and lowest re-rank score to identify the best inhibitor.

\section{ADMET studies}

The admetSAR database provides a free interface to query a distinct biological and chemical profile. The properties of ADMET profile include Adsorption, Digestion, Metabolism, Excretion, Toxicity which perform key roles in the development and discovery ofdrugs (Nayarisseri et al., 2019b; Sweta et al., 2019; Aher et al., 2019). The database preferably comprises of the 5 quantitative regression models and 22 qualitative classifications which provides the result with highly predictive precision. This estimation over properties of this database was predicted using admetSAR (http://lmmd. ecust.edu.cn:8000/). With the superior affinity of best docked virtual screened compound SCHEMBL1250485 (PubChem CID:66965667) and best-established drug Cediranib(AZD2171) PubChem CID:9933475, the bioactivity properties and toxicity was predicted by using admetSAR (Cheng et al., 2012).

\section{Drug-Likeness Prediction Studies}

A chemical inhibitor can be an effective drug if it absorbed in the required time and distributed throughout the system before getting filtered by the excretory system. In silico drug-likeness study along with ADMET has done by OSIRIS Property Explorer, that presents a group of effective parameters which help in accelerating the discovery of more effective targets and ultimately leading to drugs with the predicted biological activity which saves time and expenses (Nayarisseri et al., 2018; Sharda et al., 2019).

Web servers, Software and Suites Used

All the chemical 3D structures were retrieved from NCBI's Pubchem in SDF format and lacking 3D structure was drawn in Marvin Sketch 5.6.0.2, (1998-2011, Copyright ${ }^{\top}$ ChemAxonLtd). The ligands were optimized by using the software Schrodinger suite 2013 (Schrodinger. LLC, 2009, New York, NY). The flexible Docking was performed by taking target and all the compounds in Molegro Virtual Docker 2010.4.0.0.Molecular Visualization was done with Accelrys Discovery Studio ${ }^{\circledR}$ Visualizer 3.5.0.12158(Copyright ${ }^{\circ}$ 2005-12, Accelrys Software Inc.). ADMET profiles were studied and calculated using admetSAR (Laboratory of Molecular Modeling and Design. Copyright ${ }^{\odot} 2012$ East China University of Science and Technology, Shanghai Key Laboratory for New Drug Design).

\section{Results}

\section{Docking results}

The docking studies of complete pre-established 16 drugs resulted in Cediranib (AZD2171) (PubChem ID: 9933475) as the best-established compound (Table 2). Cediranib (AZD2171) shows the higher affinity score directed towards our target protein and has the great affinity properties as molecular weight $450.514 \mathrm{~g} / \mathrm{mol}$, hydrogen bond donor count 1 and hydrogen bond acceptor count 7, topological polar surface area $72.5 \mathrm{~A}^{2}$ and $\log \mathrm{P}$ value is 4.9. Thus the compound Cediranib has superior inhibitory affinity over protein VEGF in Glioblastoma. 
Table 2. Established Drugs Docking Result

\begin{tabular}{lcccc}
\hline $\begin{array}{l}\text { Ligand } \\
\text { PubChem ID }\end{array}$ & $\begin{array}{c}\text { MolDock } \\
\text { Score }\end{array}$ & $\begin{array}{c}\text { Rerank } \\
\text { Score }\end{array}$ & H-Bond & MW \\
\hline 9933475 & -137.596 & -108.652 & -3.00765 & 450.505 \\
9933475 & -133.384 & -106.781 & -2.68048 & 450.505 \\
9933475 & -136.24 & -102.165 & -2.64373 & 450.505 \\
9936664 & -148.731 & -101.716 & -2.97578 & 525.638 \\
10297043 & -121.812 & -93.5305 & 0.000 & 440.583 \\
5329102 & -128.833 & -93.4814 & -0.58886 & 398.474 \\
10297043 & -124.403 & -92.0401 & -0.14425 & 440.583 \\
5329102 & -129.038 & -90.9477 & -4.07277 & 398.474 \\
25102847 & -106.939 & -88.7836 & -3.54584 & 501.506 \\
\hline
\end{tabular}

\section{Virtual screened results}

Advance similarity search for the compound cediranib displayed 139 compounds. Table 3 shows the ten superior docking result of entire 139 virtual screened compounds showed SCHEMBL1250485 (PubChem CID:66965667) shown in (Figure 1) as a high affinity compound with the lowest rerank score. This compound has a molecular weight of $484.531 \mathrm{~g} / \mathrm{mol}, 1$ hydrogen bond donor, 6 hydrogen bond acceptor, a topological surface area of 74.2 $\mathrm{A}^{2}$ and a $\log \mathrm{P}$ value is 5.5. Among all these 139

Table 3. Virtual Screened Compounds Docking Result with Reference to High Affinity Cediranib (AZD2171)

\begin{tabular}{llccc}
\hline Ligand & $\begin{array}{c}\text { MolDock } \\
\text { Score }\end{array}$ & $\begin{array}{c}\text { Rerank } \\
\text { Score }\end{array}$ & H-Bond & MW \\
\hline 66965667 & -152.508 & -117.928 & -4.89163 & 484.522 \\
66965667 & -147.28 & -115.559 & 0.0000 & 484.522 \\
69090654 & -141.166 & -114.958 & -3.79463 & 478.515 \\
69090654 & -141.166 & -114.958 & -3.79463 & 478.515 \\
10196058 & -138.069 & -113.024 & -4.18063 & 511.564 \\
44156780 & -138.201 & -112.568 & -3.52407 & 450.505 \\
44156780 & -138.201 & -112.568 & -3.52407 & 450.505 \\
44156354 & -140.988 & -110.062 & -2.16748 & 450.505 \\
44156354 & -140.988 & -110.062 & -2.16748 & 450.505 \\
$1.23 \mathrm{E}+08$ & -129.443 & -109.309 & -3.53349 & 466.505 \\
\hline
\end{tabular}

compounds, the drug with PubChem CID:66965667 has much potential inhibition against Glioblastoma over the target protein VEGF.

\section{Drug-Drug comparison}

Table 3 discloses the re-rank scores of the compounds against the target protein $V E G F$ on Glioblastoma. (Table 4), the total energy of the newly found inhibitor Pubchem ID- 66965667 was lowest among the entire virtual screened compound shows its better affinity. Interestingly, the other interaction of both the compounds displaying the virtual screened compound has less affinity interaction properties according to the steric energy of PLP (Piecewise Linear Potential) but steric energy of LJ12-6 (Leonard-Jones approximation) is almost same as the pre-established Cediranib. Whereas the hydrogen bonds stability is seen more in the virtual screened drug than established inhibitor Cediranib. So-and-so, it demonstrates that the virtual screened compounds have higher potential inhibition towards the target protein $V E G F$ for Glioblastoma.

\section{Pharmacophore mapping}

Pharmacophore mapping provides a three- dimensional essential systematic topographies of molecular interaction with specific target receptors apart from the method of molecular docking. Pharmacophore studies provide an accurate query on the optimum interaction with suitable target annotations and represent the aligned poses of the molecule and help us to find the high interaction mode between the target proteinand the new compound. In spite of admirable affinity and good interaction profile of inhibitor Cediranib, Pubchem CID: 9933475 proves to have better screened result, which carries forward the study to the Pharmacophore results. Pharmacophore mapping also showed the positive intensities of electrostatics and intensities that vary in hydrophobicity, aromatic, ionizability and the distribution of interpolated charges respectively.

The residue interaction of virtually screened chemical compound SCHEMBL1250485 (Pub CID: 66965667) in

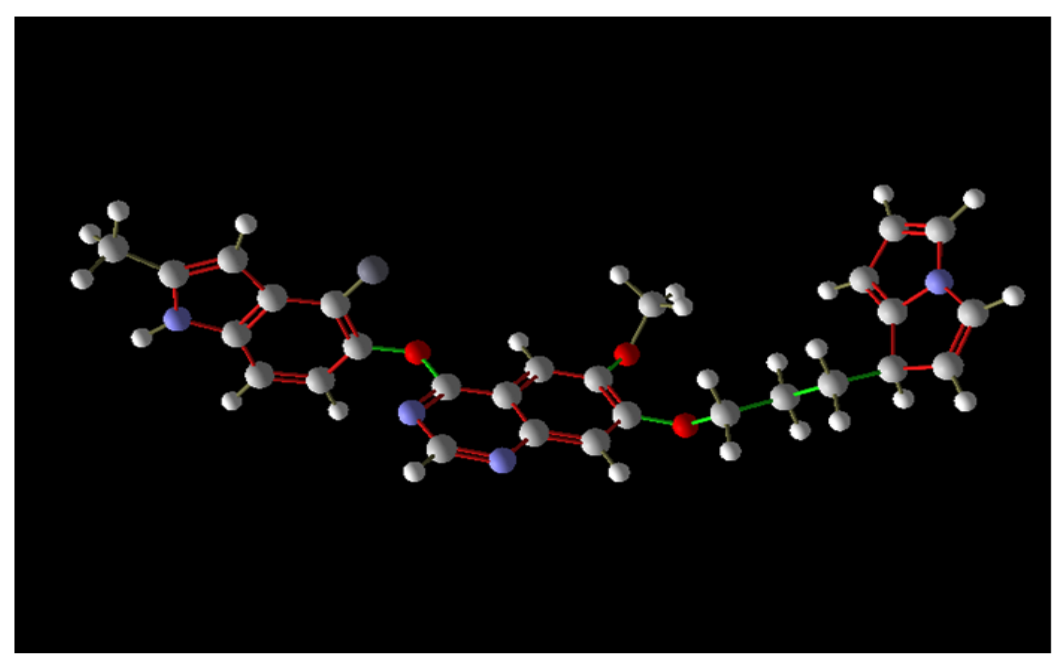

Figure 1. Best Virtual Screened Compound SCHEMBL1250485 (PubChem CID:66965667) 3D Structure Obtained from Pubchem 


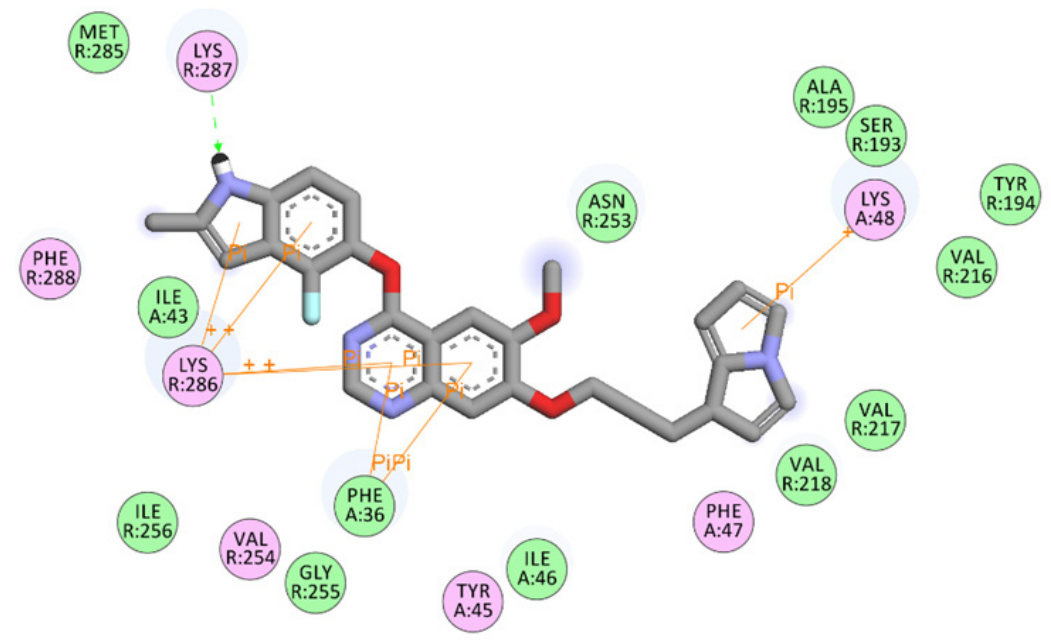

Figure 2. Screened Compound SCHEMBL1250485 (CID:66965667) Showing the Van Der Waal Interactions

the cavity of target protein VEGF (Figure 2) displayed residues with ligands where the green circled are van der Waals interaction and the pink residues circled are Electrostatic interactions. A green arrow between residues and ligand shows Hydrogen bond interaction. The virtual compound has three hydrogen bonds among which one green dotted arrow shows LYS R287 as hydrogen donor in Figure 2. Subsequently, green circles and brown

Table 4. Drug - Drug Comparative Study

\begin{tabular}{lcc}
\hline & Virtual Screened (Pubchem CID: 66965667) & Established (Cediranib Pubchem CID: 9933475) \\
\hline Energy overview:Descriptors & Rerank Score & Rerank Score \\
Total Energy & -117.927 & -108.925 \\
External Ligand interactions & -133.554 & -126.948 \\
Protein-Ligand interactions & -133.554 & -126.948 \\
Steric (by PLP) & -106.676 & -103.941 \\
Steric (by LJ12-6) & -23.001 & -23.008 \\
Hydrogen bonds & -3.876 & 0 \\
Internal Ligand interactions & 15.627 & 18.023 \\
Torsional strain & 5.386 & 2.851 \\
Torsional strain (sp2-sp2) & 0 & 0 \\
Hydrogen bonds & 0 & 0 \\
Steric (by PLP) & 0.369 & 1.738 \\
Steric (by LJ12-6) & 9.871 & 13.435 \\
Electrostatic & 0 & 0 \\
\hline
\end{tabular}

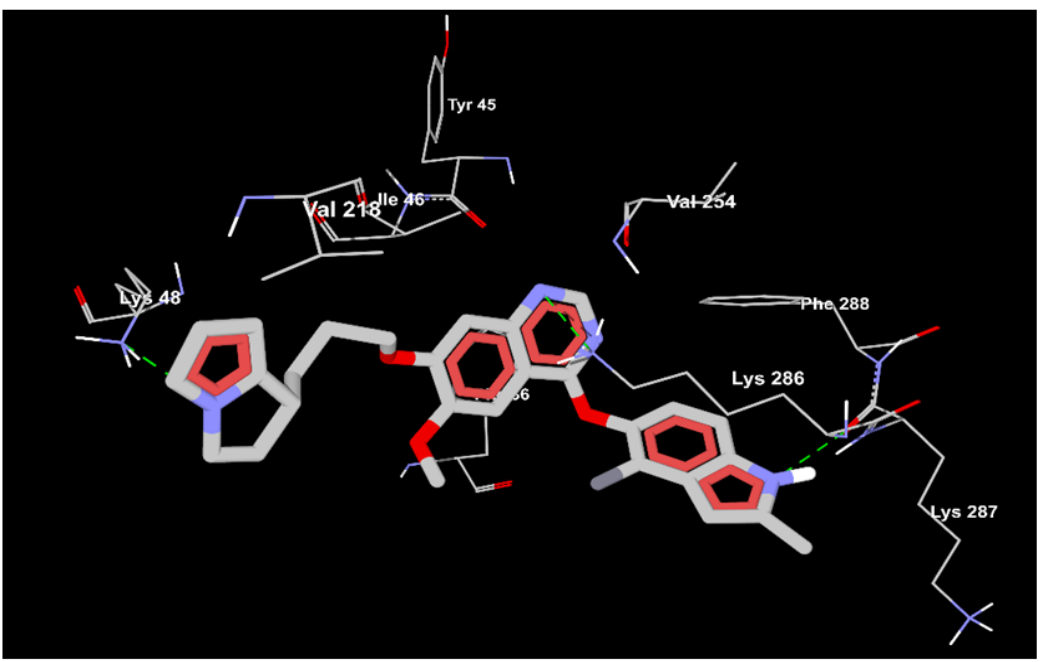

Figure 3. Screened Compound SCHEMBL1250485 (CID:66965667) Having the Hydrogen Bond Interaction 


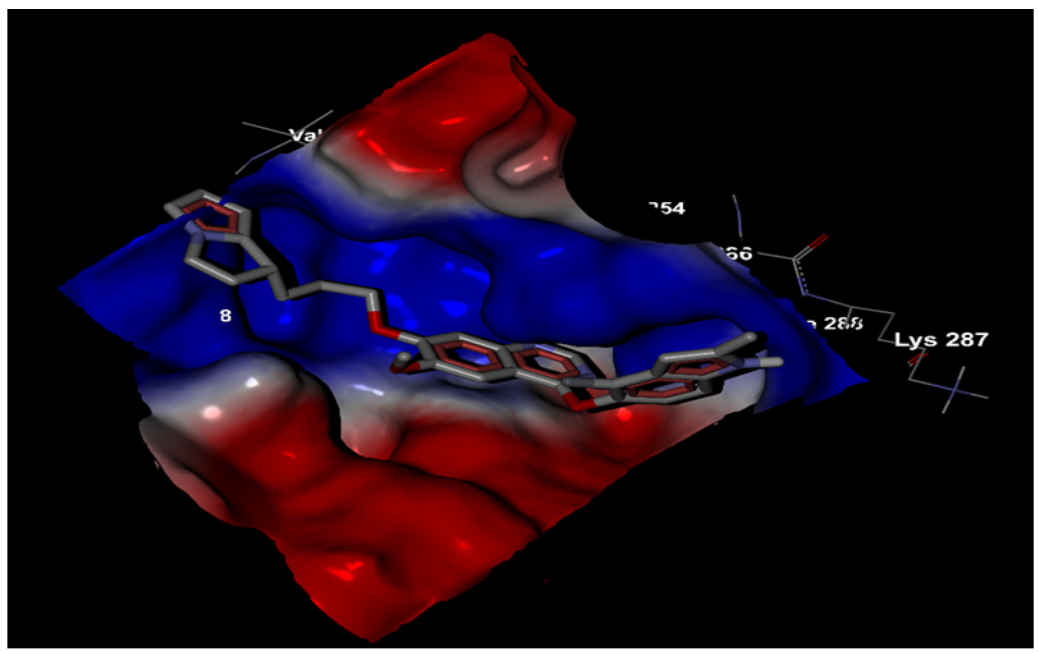

Figure 4. Screened Compound SCHEMBL1250485 (CID:66965667) Showing Electrostatic Interaction

Table 5. ADMET Profile Calculation of Both Best Docked Compound by AdmetSAR

\begin{tabular}{|c|c|c|c|c|}
\hline \multirow[t]{2}{*}{ Model } & \multicolumn{2}{|c|}{$\begin{array}{l}\text { Virtual screened compound SCHEMBL1250485 } \\
\text { (CID:66965667) }\end{array}$} & \multicolumn{2}{|c|}{$\begin{array}{l}\text { Cediranib (AZD2171) } \\
\quad \text { CID:9933475 }\end{array}$} \\
\hline & Result & Probability & Result & Probability \\
\hline \multicolumn{5}{|l|}{ Absorption } \\
\hline Blood-Brain Barrier & $\mathrm{BBB}+$ & 0.9202 & $\mathrm{BBB}+$ & 0.972 \\
\hline Human Intestinal Absorption & HIA + & 0.993 & HIA + & 0.9928 \\
\hline Caco-2 Permeability & $\mathrm{Caco} 2+$ & 0.5552 & Caco2+ & 0.5919 \\
\hline P-glycoprotein Substrate & Non-substrate & 0.6185 & Substrate & 0.6286 \\
\hline \multirow[t]{2}{*}{ P-glycoprotein Inhibitor } & Inhibitor & 0.5895 & Inhibitor & 0.7308 \\
\hline & Inhibitor & 0.6986 & Inhibitor & 0.8118 \\
\hline Renal Organic Cation Transporter & Non-inhibitor & 0.6097 & Inhibitor & 0.6936 \\
\hline \multicolumn{5}{|l|}{ Distribution } \\
\hline Subcellular localization & Mitochondria & 0.7723 & Mitochondria & 0.6861 \\
\hline \multicolumn{5}{|l|}{ Metabolism } \\
\hline CYP450 2C9 Substrate & Non-substrate & 0.7816 & Non-substrate & 0.8505 \\
\hline CYP450 2D6 Substrate & Non-substrate & 0.7468 & Non-substrate & 0.5395 \\
\hline CYP450 3A4 Substrate & Substrate & 0.7121 & Substrate & 0.7607 \\
\hline CYP450 1A2 Inhibitor & Inhibitor & 0.8174 & Inhibitor & 0.6053 \\
\hline CYP450 2C9 Inhibitor & Inhibitor & 0.5491 & Non-inhibitor & 0.5716 \\
\hline CYP450 2D6 Inhibitor & Non-inhibitor & 0.7921 & Non-inhibitor & 0.6498 \\
\hline CYP450 2C19 Inhibitor & Inhibitor & 0.7867 & Inhibitor & 0.7982 \\
\hline CYP450 3A4 Inhibitor & Inhibitor & 0.5804 & Inhibitor & 0.6267 \\
\hline CYP Inhibitory Promiscuity & $\begin{array}{l}\text { High CYP Inhibitory } \\
\text { Promiscuity }\end{array}$ & 0.9417 & $\begin{array}{l}\text { High CYP Inhibitory } \\
\text { Promiscuity }\end{array}$ & 0.922 \\
\hline \multicolumn{5}{|l|}{ Excretion Toxicity } \\
\hline \multirow{2}{*}{$\begin{array}{l}\text { Human Ether-a-go-go-Related Gene } \\
\text { Inhibition }\end{array}$} & Weak inhibitor & 0.9458 & Weak inhibitor & 0.7652 \\
\hline & Non-inhibitor & 0.5297 & Inhibitor & 0.8887 \\
\hline AMES Toxicity & Non AMES toxic & 0.5649 & Non AMES toxic & 0.641 \\
\hline Carcinogens & Non-carcinogens & 0.9335 & Non-carcinogens & 0.9197 \\
\hline Fish Toxicity & Low FHMT & 0.5698 & Low FHMT & 0.545 \\
\hline Tetrahymena Pyriformis Toxicity & High TPT & 0.9667 & High TPT & 0.8544 \\
\hline Honey Bee Toxicity & Low HBT & 0.7818 & Low HBT & 0.8482 \\
\hline Biodegradation & Not ready biodegradable & 1 & Not ready biodegradable & 1 \\
\hline Acute Oral Toxicity & III & 0.6654 & III & 0.7212 \\
\hline Carcinogenicity (Three-class) & Non-required & 0.4995 & Non-required & 0.6297 \\
\hline
\end{tabular}


Table 6. ADMET Profile- Regression

\begin{tabular}{|c|c|c|c|}
\hline & & SCHEMBL1250485 (CID:66965667) & Cediranib(AZD2171) CID:9933475 \\
\hline Model & Unit & Value & Value \\
\hline \multicolumn{4}{|l|}{ Absorption } \\
\hline Aqueous solubility & $\log S$ & -3.6396 & -3.132 \\
\hline Caco-2 Permeability & LogPapp, $\mathrm{cm} / \mathrm{s}$ & 1.1549 & 0.972 \\
\hline \multicolumn{4}{|l|}{ Toxicity } \\
\hline Rat Acute Toxicity & $\mathrm{LD} 50, \mathrm{~mol} / \mathrm{kg}$ & 2.5448 & 2.5985 \\
\hline Fish Toxicity & $\mathrm{pLC} 50, \mathrm{mg} / \mathrm{L}$ & 0.8161 & 1.2558 \\
\hline Tetrahymena Pyriformis Toxicity & 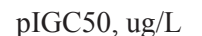 & 0.6018 & 0.4099 \\
\hline
\end{tabular}

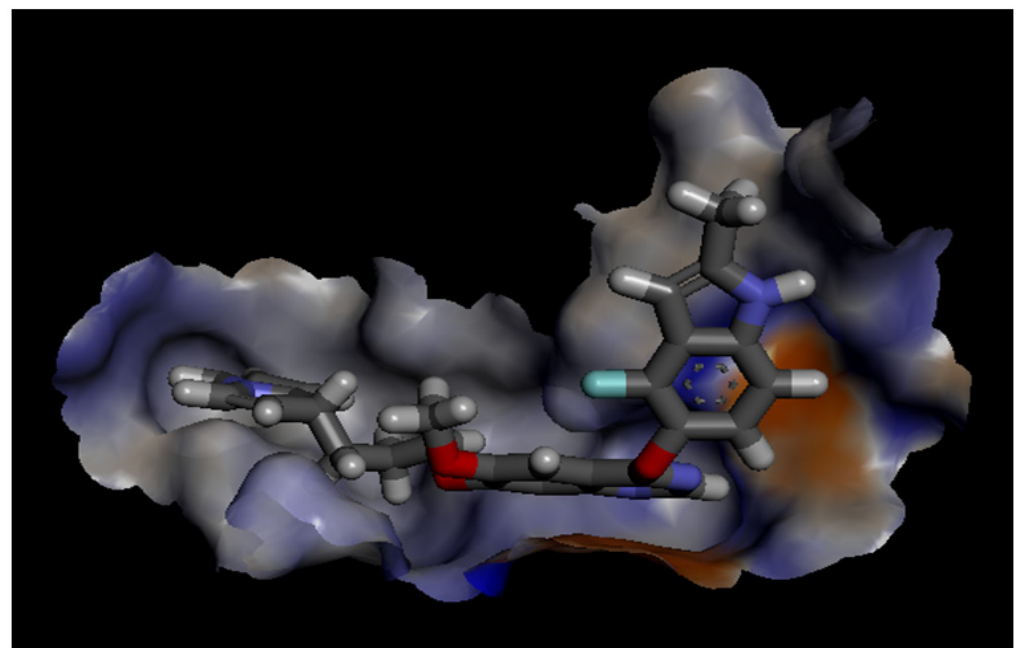

Figure 5. Screened Compound SCHEMBL1250485 (CID:66965667) Revealed the Aromatic Interaction

lines represent the van der Waal interaction and pi-pi interactions respectively. It was clear that the compound showed high Van der Waal interaction with Met R-285, Ile A-43, Ile R-256, Ile A-46, Gly R-255, Phe A-36, Asn R-253, Val R-218, Val R-217, Val R-216, Ala R-195, Ser R-193, and Tyr R-194. The compound also displays the pi-pi interaction with Lys R-286, Phe A-36, and Lys A-48.

In Figure 3 virtual screened SCHEMBL1250485 (CID:66965667) has three hydrogen bonds with Lys 48, Lys 287 and Lys 286 which are depicted by green dotted lines delineating the high affinity towards the active site of VEGF as compared to the established compound Cediranib (CID:9933475) which has zero hydrogen bond.

The electrostatic surface of the protein has two types of shaded area, the blue shaded region being the electropositive surface and the red shaded region representing the electronegative surface. The virtual screened compound SCHEMBL1250485 (CID:66965667) embedded in the VEGF protein cavity with a high-affinity shown in Figure 4. The chemical compound SCHEMBL1250485 (CID:66965667) was completely sheltered in the highly electropositive residues shown in the blue colour. Figure
5 depicts the aromatic interaction of target protein VEGF cavity against screened compound SCHEMBL1250485 (CID:66965667). The targeted protein VEGF displays the aromatic edge and face by the shades of blue and orange respectively. The molecule was showing less aromatic interaction.

\section{ADMET profile}

In Table 5, the ADMET prediction of both the best-screened compound SCHEMBL1250485 (CID:66965667) and established Cediranib (AZD2171) CID:9933475, is nearly equivalent and done with admetSAR tool. As accordingly brain penetration prediction that is BBB (Blood-Brain Barrier), cediranib displays the positive to the property of absorbing. Human Intestinal Absorption (HIA) of new drug shows a greater absorption in the intestine as the screened compound SCHEMBL1250485 (CID:66965667) as shown to have a positive tendency. The predictions on P-glycoprotein Substrate and P-glycoprotein Inhibition, Cediranib shows at a higher extent than the screened compound SCHEMBL1250485 (CID:66965667). The absorption site

Table 7. Comparative ADMET Profile with Two Test Ligands and Two Controls

\begin{tabular}{lllllll}
\hline Inhibitor name/Pub ID & BBB & HIA & CYP Substrate/inhibition & AMES toxicity & Carcinogenicity & LD50 in rat \\
\hline 66965667 & 0.9202 & 0.993 & Nonsubstrate/inhibitor & 0.5649 & Non-carcinogens & 2.5448 \\
69090654 & 0.9401 & 0.9853 & Nonsubstrate/noninhibitor & 0.6817 & Non-carcinogens & 2.5589 \\
Cediranib & 0.972 & 0.9928 & Nonsubstrate/inhibitor & 0.641 & Non-carcinogens & 2.5985 \\
9936664 & 0.842 & 0.9824 & Nonsubstrate/noninhibitor & 0.7183 & Non-carcinogens & 2.5968 \\
\hline
\end{tabular}




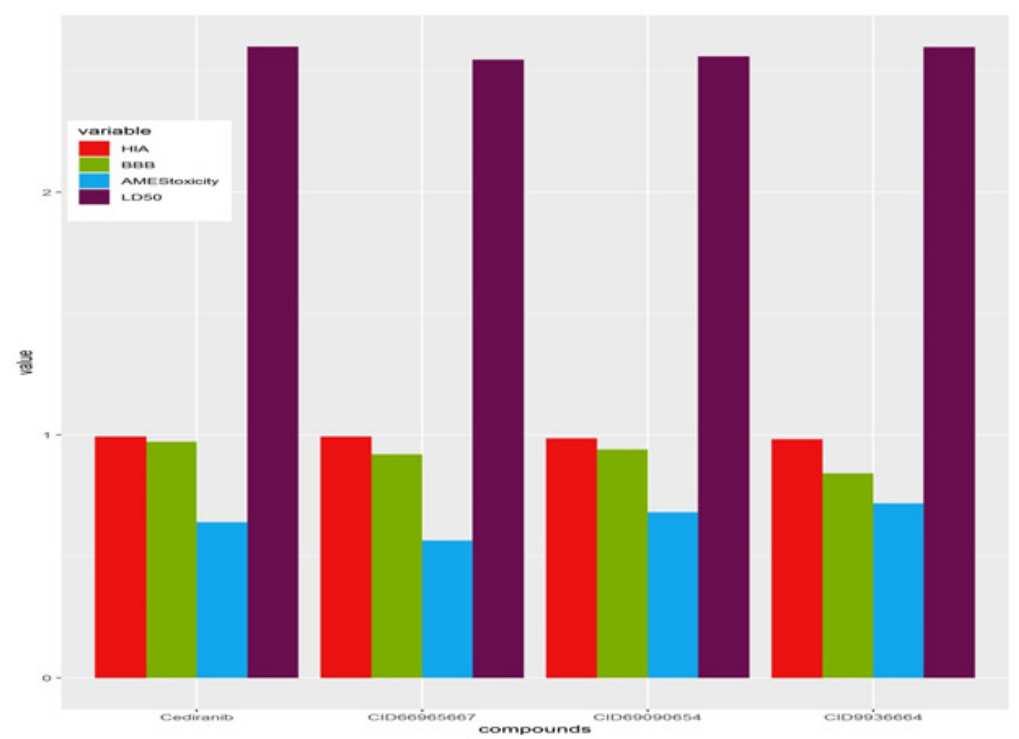

Figure 6. Comparative ADMET Studies of BBB, HIA, AMES Toxicity and LD50 of the Established Compounds (cediranib, PubID9936664) against Virtual Screened Compounds

Table 8. Drug-Likeness Prediction Through OSIRIS Property Explorer

\begin{tabular}{lccccc}
\hline S.N & Inhibitor & clogP & Log S & Molecular weight & TPSA \\
\hline 1 & PubCID 66965667 & 5.92 & -7.38 & 484 & 74.1 \\
2 & PubCID 69090654 & 4.61 & -6.81 & 478 & 89.57 \\
3 & Cediranib & 4.5 & -6.56 & 450 & 72.5 \\
4 & PubID9936664 & 4.24 & -6.25 & 525 & 72.8 \\
\hline
\end{tabular}

for the P-glycoprotein substrate for the screened compound SCHEMBL1250485 (CID:66965667) reveals it has less probability than established Cediranib (CID:9933475) as similarly P-glycoprotein Inhibitor shows the values with low probability.

In addition to the distributionof subcellular localization in both the established and screened compounds in the mitochondria, the screened compound (CID:66965667) shows the distribution to have a high probability than others. In the case of metabolism, they vary in some points like CYP450 3A4, CYP450 2C19 Inhibitor, and CYP450 1A2 Inhibitor where both the compounds are acting as substrate as well as the inhibitors. CYP450 $2 \mathrm{C} 9$ acts as non-inhibitor for cediranib. Overall both the compounds display the equivalent high inhibitory effect towards the target protein. Further study of bioactivity in the profile of excretion and toxicity is almost equivalent, but in reference to Carcinogens they vary as the virtual screened compound is shown to have high amount of non-carcinogens than established docked compound. The compounds are mutagenic or not that can predict by ADMET regression toxicity study. Both the compounds in the properties of Rat Acute Toxicity are nearly equal to each other. But the possibility of having higher toxicity than these two molecules is shown in (Table 6). Additional study of bioactivity in the profile of excretion and toxicity shows it to be almost equivalent, but in reference to fish toxicity, they vary as Cediranib shows high toxicity than

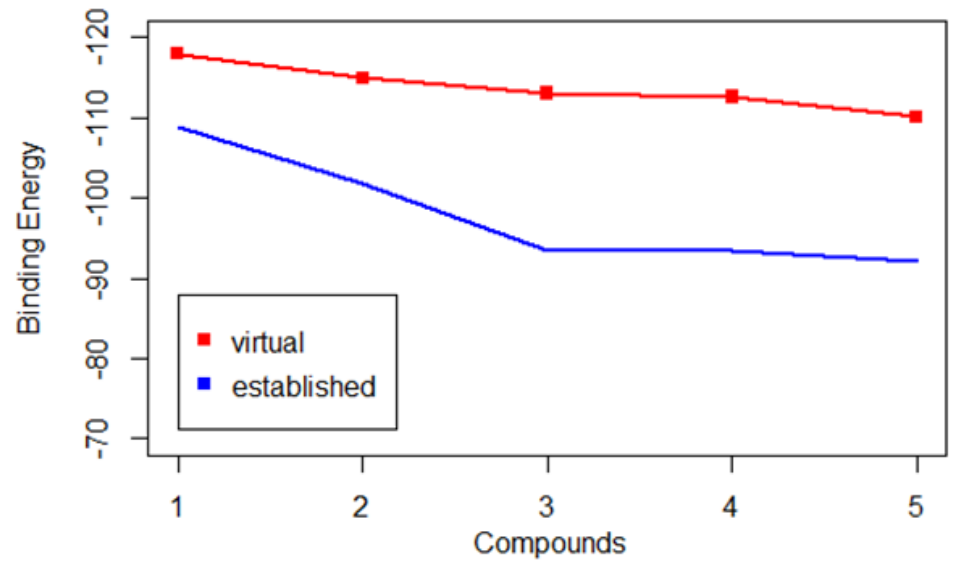

Figure 7. Drug-Likeness Study with Five Best Virtually Screened Compound and Five Best Established Inhibitors by Using OSIRIS Property Explorer Software 
the virtual screened compound.

Comparative ADMET profile study of the compounds and the control

The comparative ADMET profile for the established and predicted inhibitors based on important parameters such as BBB (Blood-Brain Barrier), HIA (Human Intestinal Absorption), AMES toxicity, and LD50. Preferably four best chemical compounds, the best virtual screened inhibitor Pubchem ID-66965667 and established drug cediranib with the two other Pubchem CID:69090654 and Pubchem CID:9936664 has been taken for the comparative ADMET studies.

These four compounds were graphically projected using R-programming as revealed down in the (Figure 6). The parameters such as BBB, HIA, AMES Toxicity, and LD50 were obtained from the admetSAR database and were tabulated according to their properties and predicted values. As shown in the graph as well as tabular data (Table 7) among the four compounds the values of BBB were higher in the established drug cediranib and then the second best virtual screened compound with PubChem ID-69090654 as comparative to other compounds. Human Intestinal Absorption (HIA) of the best virtual drug (CID:66965667) compounds is somewhat higher than the cediranib. AMES toxicity is lowest for the virtual drug CID:69090654, among the best four Compounds. The LD50 is almost alike for all the four compounds.

\section{Drug-Likeness Prediction Studies}

Table 7 depicts the drug-likeness properties of two best virtually docked compounds and two best-established compounds using OSIRIS Property Explorer. Although clogP-value which stands for an algorithm of compound's partition coefficient between N-octanol and water and molecular weight for an algorithm virtual screened compound (PubCID 69090654) have similar absorption property as the best established compound cediranib but $\log \mathrm{S}$ values clarify that solubility of the best virtual screened compound (PubCID 66965667) was highest among best four. TPSA belonging to polar atoms in the compound showing less difference in the values of best virtually screened compound (PubCID 66965667) and the best-established drug cediranib. Beside all the properties, Figure 7 clearly shows the binding energy of the best virtual screened compound (PubCID 66965667) (-117.928) with the target $V E G F$ was quite lesser than the best-established compound cediranib $(-108.925)$ the property was proving that affinity of the newly screened compound for target protein $V E G F$ in GBM was higher.

\section{Discussion}

Being the most aggressive tumor in a human, Glioblastoma drew the noteworthy attention of scientists and researchers all over the world. According to the research in spite of having various treatments for glioblastoma including chemotherapy, radiation therapy, surgery, a diagnosed patient does not have long-term survival rate and also have numerous side effects (Nayarisseri et al., 2018; Sharda et al., 2019). In the present study, we proposed a newly screened inhibitor which directly can block the active site of VEGFR and have found to be less toxicity and cytotoxicity on ADMET analysis. Among the 16 established inhibitors from various studies that inhibit VEGF activity, Cediranib (Batchelor et al., 2007b), CEP-7055 (Jones-Bolin et al., 2006b) found to be best best known for the treatment of glioblastoma. In present study, we found an effective inhibitor SCHEMBL1250485 (PubChem CID: 66965667) by the mean of In silico-approaches.

The ranking and evaluation of the predicted inhibitor conformations is a critical aspect of structure-based virtual screening. However, estimations of structure-based screening parameters have yielded impressive results and numerous novel hits (Kitchen et al., 2004). Virtual screened inhibitor SCHEMBL1250485 have lower rerank score $(-117.927)$ than the pre-established inhibitors which give the better affinity to the ligand to bind with the active site. SCHEMBL1250485 has a low probability of absorption for blood Brain barrier which is an essential property for a drug. Established inhibitor cediranib has a comparatively high chance for the same. SCHEMBL1250485 has low AMES toxicity as well as carcinogenicity than the best-established inhibitor cediranib. As SCHEMBL1250485 has better and enhanced properties as a suitable inhibitor of VEGFR in glioblastoma, our findings suggest the better active site inhibitor which can further be used for the In vitro studies and drug development.

In conclusion, Glioblastoma multiforme (GBM) is a fast-growing glioma that is developed from astrocytes and oligodendrocytes leads to devastating brain cancer, always leading to mortality within the span of a year. Vascular endothelial growth factor $(V E G F)$ is the most abundant and essential mediator of angiogenesis in glioblastoma as it promotes proliferation. In this present research, an attempt has been made to demonstrate from the study of pre-existing inhibitors found against inhibitory protein $V E G F$ which in turn help to inhibit Glioblastoma. The computational prediction of 16 pre-established drugs such as molecular docking studies followed by virtual screening and comparative studies of the best drugs from docking result and virtual docked result, we found the inhibitorCID:66965667has admirable properties to inhibit VEGF. Additionally, the best-established docked compound Cediranib (AZD2171) has PubChem CID:9933475 and the virtual screening resulted in compound PubChem CID:66965667 examine for ADMET study in terms of toxicity as a non-carcinogenic and nonmutagenic profile where both the perspectives reveal analogous properties, somehow PubChem CID:9933475 was found having higher probability in terms of BBBBlood Brain Barrier, whereas HIA-Human Intestinal Absorption, AMES toxicity, and LD50 is having higher probability. In spite of radio-chemotherapy and advances in surgery, glioblastoma still shows a lot of resistance towards treatment and PubChem CID:66965667 medicines. In this scenario, new approaches to study glioblastoma and to design optimized therapies are greatly needed. The current study radicalised the potential inhibitor SCHEMBL1250485 endowed by ADMET and 
drug-likeness study with the enhanced binding affinity, therapeutic properties, less toxicity than established drug Cediranib. As per the comparative virtual screened drug properties with all prediction as a potential candidate, it could be also optimized for a remarkable pharmacological profile. Hence the study ahead on these compounds may be subjected to In vitro analysis for its pharmacokinetic and biological activity.

\section{Acknowledgements}

SKS thank Department of Biotechnology (DBT), New Delhi (No. BT/PR8138/BID/7/458/2013, dated 23rd May, 2013), DST-PURSE $2^{\text {nd }}$ Phase Programme Order No. SR/PURSE Phase 2/38 (G dated 21.02.2017 and FIST (SR/FST/LSI - 667/2016), MHRD RUSA - Phase 2.0 (grant sanctioned vide letter No. F. 24-51 / 2014 - U, Policy (TNMulti-Gen), Dept. of Edn. Govt. of India, Dt. 09.10.2018) for providing the financial assistance.

\section{Competing interests}

The authors declare that they have no competing interests.

\section{Consent for publication \\ Not applicable.}

\section{Ethics approval and consent to participate} Not applicable.

\section{References}

Aher A, Udhwani T, Khandelwal R, et al (2019). In silico insights on IL-6: A potential target for multicentric castleman disease. Curr Comput Aided Drug Des, PMID:31475901

Akare, U.R, Bandaru, S, Shaheen, U, et al (2014). Molecular docking approaches in identification of High affinity inhibitors of Human SMO receptor. Bioinformation, 10, 737-42.

Ali MA, Vuree S, Goud H, et al (2019). Identification of high-affinity small molecules targeting gamma secretase for the treatment of Alzheimer's disease. Curr Top Med Chem, 19, 1173-1187.

Babitha PP, Sahila MM, Bandaru S, et al (2015). Molecular docking and pharmacological investigations of rivastigminefluoxetine and coumarin-tacrine hybrids against acetyl choline esterase. Bioinformation, 11, 378-86.

Babitha PP, Sahila MM, Srinivas B, et al (2017). Common SAR derived from linear and non-linear QSAR studies on AChE inhibitors used in the treatment of Alzheimer's disease. Curr Neuropharmacol, 15, 1093 - 9.

Bandaru S, Alvala M, Nayarisseri A, et al (2017a). Molecular dynamic simulations reveal suboptimal binding of salbutamol in T164I variant of beta2 adrenergic receptor. PLoS One, 12, e0186666.

Bandaru S, Marri VK, Kasera P, et al (2014). Structure based virtual screening of ligands to identify cysteinyl leukotriene receptor 1 antagonist. Bioinformation, 10, 652-7.

Bandaru S, Ponnala D, Lakkaraju C, et al (2015a). Identification of high affinity non-peptidic small molecule inhibitors of MDM2-p53 interactions through structure-based virtual screening strategies. Asian Pac J Cancer Prev, 16, 3759-65.

Bandaru S, Prasad MH, Jyothy A, et al (2013). Binding modes and pharmacophoric features of muscarinic antagonism and beta2 agonism (MABA) conjugates. Curr Top Med Chem, 13, 1650-5.

Bandaru S, Sumithnath TG, Sharda S, et al (2017b). Helix-Coil transition signatures $B$-Raf V600E mutation and virtual screening for inhibitors directed against mutant $B$-Raf. Curr Drug Metab, 18, 527-34.

Bandaru S, Tiwari G, Akka J, et al (2015b). Identification of high affinity bioactive Salbutamol conformer directed against mutated (Thr164Ile) beta 2 adrenergic receptor. Curr Top Med Chem, 15, 50-6.

Bandaru S, Tarigopula P, Akka J, et al (2016). Association of Beta 2 adrenergic receptor (Thr164Ile) polymorphism with Salbutamol refractoriness in severe asthmatics from Indian population. Gene, 592, 15-22.

Basak SC, Nayarisseri A, Gonzalez-Diaz H, et al (2016a). Editorial (Thematic Issue: Chemoinformatics Models for Pharmaceutical Design, Part 1). Curr Pharm Des, 22, 5041-2.

Basak SC, Nayarisseri A, Gonzalez-Diaz H, et al (2016b). Editorial (Thematic Issue: Chemoinformatics Models for Pharmaceutical Design, Part 2). Curr Pharm Des, 22, 5177-8.

Batchelor TT, Sorensen AG, di Tomaso E, et al (2007b). AZD2171, a pan-VEGF receptor tyrosine kinase inhibitor, normalizes tumor vasculature and alleviates edema in glioblastoma patients. Cancer Cell, 11, 83-95.

Batchelor T, Sorensen AG, Ancukiewicz M, et al (2007a). A phase II trial of AZD2171 (cediranib), an oral pan-VEGF receptor tyrosine kinase inhibitor, in patients with recurrent glioblastoma. J Clin Onco, 25, 2001.

Brozzo MS, Bjelić S, Kisko K, et al (2012). Thermodynamic and structuraldescriptionofallosterically regulated VEGFR-2 dimerization. Blood, 119, 1781-8.

Chandrakar B, Jain A, Roy S, et al (2013). Molecular modeling of Acetyl-CoA carboxylase (ACC) from Jatropha curcas and virtual screening for identification of inhibitors. $J$ Pharm Res, 6, 913-8.

Cheng F, Li, W, Zhou Y, et al (2012). AdmetSAR, a comprehensive source and freetoolforassessment of chemical ADMET properties. J Chem Inf Model, 52, 3099-3105.

Divya Jain TU, Shreshtha S, Aishwarya G, et al (2019). Design of novel JAK3 inhibitors towards Rheumatoid Arthritis using molecular docking analysis. Bioinformation, 15, 68-78.

Dunna NR, Bandaru S, Raj Akare U, et al (2015a). Multiclass comparative virtual screening to identify novel Hsp90 inhibitors: a therapeutic breast cancer drug target. Curr Top Med Chem, 15, 57-64.

Dunna NR, Kandula V, Girdhar A, et al (2015b). High affinity pharmacological profiling of dual inhibitors targeting RET and VEGFR2 in inhibition of kinase and angiogeneis events in medullary thyroid carcinoma. Asian Pac J Cancer Prev, 16, 7089-95.

Ferrara N, Gerber HP, LeCouter J, et al (2003). The biology of VEGF and its receptors. Nat Med, 9, 6766-9.

Folkman J (1971). Tumor angiogenesis, therapeutic implications. N Engl J Med, 285, 1182-6.

Gerstner ER, Sorensen AG, Jain RK, et al (2009). Anti-vascular endothelial growth factor therapy for malignant glioma. Curr Neurol Neurosci Rep, 9, 254-62.

Greenberg DA, Jin K (2005). From angiogenesis to neuropathology. Nature, 438, 954-9.

Gudala S, Khan U, Kanungo N, et al (2015). Identification and pharmacological analysis of high efficacy small molecule inhibitors of EGF-EGFR interactions in clinical treatment of non-small cell lung carcinoma: A computational approach. Asian Pac J Cancer Prev, 16, 8191-6.

Gutlapalli VR, Sykam A, Nayarisseri A, et al (2015). 
Insights from the predicted epitope similarity between Mycobacterium tuberculosis virulent factors and its human homologs. Bioinformation, 11, 517-24.

Hanahan D, Folkman J (1996). Patterns and emerging mechanisms of the angiogenic switch during tumorigenesis. Cell, 86, 353-64.

Hernandez-Pedro N, Rangel-Lopez E, Vargas Félix G, Pineda B, Sotelo J (2013). An update in the use of antibodies to treat glioblastoma multiforme. Autoimmune Dis, 2013, 716813.

Holland CE (2000). Glioblastoma multiforme: the terminator. Proc Natl Acad Sci U S A, 97, 6242-6.

Jawhari S, Ratinaud MH1, Verdier M1 (2016). Glioblastoma, hypoxia and autophagy: a survival-prone 'menage-a-trois' . Cell Death Dis, 7, e2434.

Jones-Bolin S, Zhao H, Hunter K, Klein-Szanto A, Ruggeri B (2006a). The effects of the oral, pan-VEGF-R kinase inhibitor CEP-7055 and chemotherapy in orthotopic models of glioblastoma and colon carcinoma in mice. Mol Cancer Ther, 5, 1744-53.

Jones-Bolin S, Zhao H, Hunter K, et al (2006b). The effects of the oral, pan-VEGF-R kinase inhibitor CEP-7055 and chemotherapy in orthotopic models of glioblastoma and colon carcinoma in mice. Mol Cancer Ther, 5, 1744-53.

Kast RE, Boockvar JA, Bruning A, et al (2013). A conceptually new treatment approach for relapsed glioblastoma: coordinated undermining of survival paths with nine repurposed drugs (CUSP9) by the International Initiative for Accelerated Improvement of Glioblastoma Care. Oncotarget, 4, 502-30.

Kelotra A, Gokhale SM, Kelotra S, et al (2014a). Alkyloxy carbonyl modified hexapeptides as a high affinity compounds for Wnt5A protein in the treatment of psoriasis. Bioinformation, 10, 743-9.

Kelotra S, Jain M, Kelotra A, et al (2014b). An in silico appraisal to identify high affinity anti-apoptotic synthetic tetrapeptide inhibitors targeting the mammalian caspase 3 enzyme. Asian Pac J Cancer Prev, 15, 10137-42.

Khandekar N, Singh S, Shukla R, et al (2016). Structural basis for the in vitro known acyl-depsipeptide 2 (ADEP2) inhibition to Clp 2 protease from Mycobacterium tuberculosis. Bioinformation, 12, 92-7.

Khandelwal R, Chauhan A, Hussain T, et al (2018). Structure-based virtual screening for the identification of high affinity small molecule towards STAT3 for the clinical treatment of Osteosarcoma. Curr Top Med Chem, 18, 2511-26.

Kitchen DB, Decornez H, Furr JR, Bajorath J (2004). Docking and scoring in virtual screening for drug discovery, methods and applications. Nat Rev Drug Discov, 3, 935-49.

Lee DH, Ryu HW, Won HR, Kwon SH (2017). Advances in epigenetic glioblastoma therapy. Oncotarget, 8, 18577-89.

Loges S, Mazzone M, Hohensinner P, Carmeliet P (2009). Silencing or fueling metastasis with VEGF inhibitors: antiangiogenesis revisited. Cancer Cell, 15, 167-70.

Majhi M, Ali MA, Limaye A, et al (2018). An in silico investigation of potential EGFR inhibitors for the clinical treatment of colorectal cancer. Curr Top Med Chem, 18, 2355-66.

Mamluk R, Carvajal IM, Morse BA, et al (2010). Anti-tumor effect of CT-322 as an adnectin inhibitor of vascular endothelial growth factor receptor-2. MAbs, 2, 199-208.

Mangraviti A, Raghavan T, Volpin F et al (2017). HIF-1alphatargeting acriflavine provides long term survival and radiological tumor response in brain cancer therapy. Sci Rep, 7, 14978.

Mehta M, Brem S (2014). Recent updates in the treatment of glioblastoma: introduction. Semin Oncol, 41, 1-3.
Mendonça-Junior FJ, Scotti MT, Nayarisseri A, et al (2019). Natural bioactive products with antioxidant properties useful in neurodegenerative diseases. Oxid Med Cell Longev, 2019, 1-2.

Monteiro AFM, Viana JDO, Nayarisseri A, et al (2018). Computational studies applied to flavonoids against Alzheimer's and Parkinson's diseases. Oxid Med Cell Longev, 2018, 1-21.

Nasr AB, Ponnala D, Sagurthi SR, et al (2015). Molecular Docking studies of FKBP12-mTOR inhibitors using binding predictions. Bioinformation, 11, 307.

Natchimuthu V, Bandaru S, Nayarisseri A, Ravi S (2016). Design, synthesis and computational evaluation of a novel intermediate salt of N-cyclohexyl-N-(cyclohexylcarbamoyl)4-(trifluoromethyl) benzamide as potential potassium channel blocker in epileptic paroxysmal seizures. Comput Biol Chem, 64, 64-73.

Nayarisseri A (2019b). Prospects of utilizing computational techniques for the treatment of human diseases. Curr Top Med Chem, 19, 1071-4.

Nayarisseri A, Hood EA (2018). Advancement in microbial cheminformatics. Curr Top Med Chem, 18, 2459-61.

Nayarisseri,A, Yadav M (2015). Editorial (Thematic Issue: Mechanistics in drug design-experimental molecular biology vs. molecular modeling). Curr Top Med Chem, 15, 3-4.

Omuro A, DeAngelis LM (2013). Glioblastoma and other malignant gliomas: a clinical review. JAMA, 310, 1842-50.

Padmini Gokhale APSC, Anushka A, Natasha K, et al (2019). FLT3 inhibitor design using molecular docking based virtual screening for acute myeloid leukemia. Bioinformation, 15, 104-15.

Palak S, Ravina K, Diksha S, et al (2019). Virtual screening of IL-6 inhibitors for idiopathic Arthritis. Bioinformation, 15, 121-30.

Patidar K, Deshmukh A, Bandaru S (2016). Virtual screening approaches in identification of bioactive compounds Akin to delphinidin as potential HER2 inhibitors for the treatment of breast cancer. Asian Pac J Cancer Prev, 17, 2291-5.

Patidar K, Panwar U, Vuree S, et al (2019). An in silico approach to identify high affinity small molecule targeting m-TOR inhibitors for the clinical treatment of breast cancer. Asian Pac J Cancer Prev, 20, 1229-41.

Pore N, Gupta AK, Cerniglia GJ, Maity A (2006). HIV protease inhibitors decrease VEGF/HIF-1alpha expression and angiogenesis in glioblastoma cells. Neoplasia, 8, 889-95.

Praseetha S, Bandaru S, Nayarisseri A, et al (2016a). Pharmacological analysis of vorinostat analogues as potential anti-tumor agents targeting human histone deacetylases: an epigenetic treatment stratagem for cancers. Asian Pac J Cancer Prev, 17, 1571-6.

Praseetha S, Bandaru S, Yadav M, et al (2016b). Common SAR derived from multiple QSAR models on Vorinostat derivatives targeting HDACs in tumor treatment. Curr Pharm Des, 22, 5072-8.

Rao DM, Nayarisseri A, Yadav M, et al (2010). Comparative modeling of methylentetrahydrofolate reductase (MTHFR) enzyme and its mutational assessment: in silico approach. Int J Bioinformatics Res, 2, 5-9.

Reardon DA, Conrad CA, Cloughesy T et al (2012). Phase I study of AEE788, a novel multitarget inhibitor of ErbBand VEGF-receptor-family tyrosine kinases, in recurrent glioblastoma patients. Cancer Chemother Pharmacol, 69, 1507-18.

Rosell R, de Las Penas R, Balana C, et al (2008). Translational research in glioblastoma multiforme: molecular criteria for patient selection. Future Oncol, 4, 219-28.

Sahila MM, Babitha PP, Anitha J, et al (2017). Development of 
MLR and SVM aided QSAR models to identify common SAR of GABA uptake herbal inhibitors used in the treatment of Schizophrenia. Curr Neuropharmacol, 15, 1085 - 92.

Sahila MM, Babitha PP, Bandaru S, Nayarisseri A, Doss VA (2015). Molecular docking based screening of GABA (A) receptor inhibitors from plant derivatives. Bioinformation, 11, 280-9.

Sawa H, Murakami H, Ohshima Y, et al (2002). Histone deacetylase inhibitors such as sodium butyrate and trichostatin A inhibit vascular endothelial growth factor (VEGF) secretion from human glioblastoma cells. Brain Tumor Pathol, 19, 77-81.

Schwartzbaum JA, Fisher JL, Aldape KD, Wrensch M (2006). Epidemiology and molecular pathology of glioma. Nat Clin Pract Neurol, 2, 494-503.

Shaheen U, Akka J, Hinore JS, et al (2015). Computer aided identification of sodium channel blockers in the clinical treatment of epilepsy using molecular docking tools. Bioinformation, 11, 131-7.

Shameer K, Nayarisseri A, Duran FXR, et al (2017). Improving neuropharmacology using big data, machine learning and computational algorithms. Curr Neuropharmacol, 15, 1058-61.

Sharda S, Sarmandal P, Cherukommu S, et al (2017). A virtual screening approach for the identification of high affinity small molecules targeting BCR-ABL1 inhibitors for the treatment of chronic myeloid leukemia. Curr Top Med Chem, 17, 2989-96.

Sharda S, Khandelwal R, Adhikary R, et al (2019). A computeraided drug designing for pharmacological inhibition of ALK inhibitors induces apoptosis and differentiation in Non-small cell lung cancer. Curr Top Med Chem, 19, 1129-44.

Sharma K, Patidar K, Ali MA, et al (2018). Structure-based virtual screening for the identification of high affinity compounds as potent VEGFR2 inhibitors for the treatment of renal cell carcinoma. Curr Top Med Chem, 18, 2174-85.

Simpson WJ, Platts ME (1976). Fractionation study in the treatment of glioblastoma multiforme. Int J Radiat Oncol Biol Phys, 1, 639-44.

Singh SK, Nayarisseri A (2019). Functional Inhibition of VEGF and EGFR suppressors in cancer treatment. Curr Top Med Chem, 19, 178-9.

Sinha C, Nischal A, Bandaru S, et al (2015). An In silico approach for identification of novel inhibitors as a potential therapeutics targeting HIV-1 viral infectivity factor. Curr Top Med Chem, 15, 65-72.

Sinha C, Nischal A, Pant KK, et al (2014). Molecular docking analysis of RN18 and VEC5 in A3G-Vif inhibition. Bioinformation, 10, 611.

Sinha K, Majhi M, Thakur G, et al (2018). Computer aided Drug Designing for the identification of high affinity small molecule targeting CD20 for the clinical treatment of Chronic Lymphocytic Leukemia (CLL). Curr Top Med Chem, 18, 2527-42.

Stupp R, Hegi ME, Mason WP, et al (2009). Effects of radiotherapy with concomitant and adjuvant temozolomide versus radiotherapy alone on survival in glioblastoma in a randomised phase III study: 5-year analysis of the EORTC-NCIC trial. Lancet Oncol, 10, 459-66.

Sweta J, Khandelwal R, Srinitha S, et al (2019). Identification of high-affinity small molecule targeting IDH2 for the clinical treatment of acute myeloid leukemia. Asian Pac J Cancer Prev, 20, 2287-97.

Taylor J, Gerstner ER (2013). Anti-angiogenic therapy in high-grade glioma (treatment and toxicity). Curr Treat Options Neurol, 15, 328-37.

Trishang Udhwani SM, Khushboo S, Jajoriya S, et al (2019).
Design of PD-L1 inhibitors for lung cancer. Bioinformation, 15, 139-50.

Vajkoczy P, Menger MD, Vollmar B, et al (1999). Inhibition of tumor growth, angiogenesis, and microcirculation by the novel Flk-1 inhibitor SU5416 as assessed by intravital multi-fluorescence videomicroscopy. Neoplasia, 1, 31-41.

Vuree S, Dunna NR, Khan IA, et al (2013). Pharmacogenomics of drug resistance in Breast Cancer Resistance Protein (BCRP) and its mutated variants. J Pharm Res, 6, 791-8.

Zhang Y, Guessous F, Kofman A, et al (2010). XL-184, a MET, VEGFR-2 and RET kinase inhibitor for the treatment of thyroid cancer, glioblastoma multiforme and NSCLC. IDrugs, 13, 112-21.

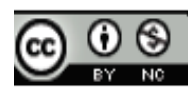

This work is licensed under a Creative Commons AttributionNon Commercial 4.0 International License. 